>Le génome de Tribolium castaneum, publié récemment, ne serait-il qu'une goutte de plus versée dans l'océan génomique? Non, car Tribolium se distingue à plus d'un titre. D'abord, en tant que coléoptère, il représente un ordre qui compte environ le quart des espèces animales. De plus, cet insecte omnivore est un nuisible pour les grains et les farines du monde entier. Enfin, Tribolium est discrètement entré dans l'élite des organismes modèles de la génétique. Cet article vise à montrer que Tribolium est un compagnon de laboratoire complémentaire de la drosophile, notamment pour la génétique du développement. <

\section{Quand Tribolium complémente la génétique de la drosophile}

\author{
François Bonneton
}

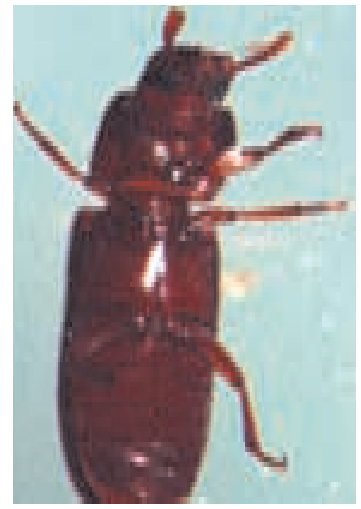

Université de Lyon, Université Lyon 1, ENS de Lyon, IGFL, CNRS UMR 5242, INRA UMR1237, 46, allée d'Italie, 69364 Lyon Cedex 07, France. francois.bonneton@ens-lyon.fr

\section{Qui est Tribolium?}

Le Tribolium rouge de la farine (Tribolium castaneum, Herbst ${ }^{1} 1797$ [1]) est un insecte de 3-4 mm de long (Figure 1) facile à élever, avec un cycle de développement court de 30 jours, une longévité de six mois à quatre ans, ce qui est exceptionnel pour un insecte (un mois pour la drosophile) et une fécondité élevée (Tableau I). Ces caractéristiques expliquent que, dès les années 1920-1930, Tribolium est devenu un organisme modèle en génétique et en biologie des populations [3].

Tribolium est un nuisible cosmopolite et polyphage dont les souillures corrompent de très nombreuses denrées amylacées, notamment les farines de céréales. Dans la nature, cet insecte vit sous l'écorce des arbres, mais les lignées de laboratoire proviennent de minoteries et de silos. Comme Tribolium est capable de résister à toutes les classes d'insecticides, le contrôle de ce ravageur nécessite de nouvelles stratégies de lutte. Le séquençage du génome [2] a justement permis d'identifier des protéines susceptibles d'être ciblées par de nouveaux insecticides, comme des canaux ioniques, des récepteurs nucléaires, des récepteurs couplés à des protéines $G(G P C R)$ et les cytochromes $P 450$, une famille d'enzymes de détoxication

${ }^{1}$ Herbst : naturaliste allemand (1743-1807). qui a subi une forte amplification chez Tribolium, avec 134 gènes contre 87 chez la drosophile [1].

Tribolium est aujourd'hui tellement cosmopolite et commensal de l'homme que son origine est incertaine. II proviendrait de régions d'Asie méridionale au climat chaud et sec, peut-être d'Inde. Le genre Tribolium comprend 36 espèces, dont quatre sont cosmopolites [4]. Ces insectes font partie de l'immense famille des Tenebrionidae, dont les 17000 espèces sont réparties dans le monde entier, de préférence dans des zones arides. Le génome de Tribolium est le premier séquencé pour un coléoptère, qui est le plus grand ordre d'insectes en nombre d'espèces, avec 350000 espèces décrites (un quart des espèces animales). Cette radiation évolutive, commencée il y a 285 millions d'années, s'est accompagnée d'une grande diversité morphologique, comportementale et écologique [5]. Le génome de Tribolium comble ainsi un manque de taille, en nombre d'espèces, pour comprendre la biodiversité. Une des principales surprises de son analyse fut de révéler une forte diversification des récepteurs olfactifs et gustatifs, qui sont trois à quatre fois plus nombreux que chez les autres insectes. Cette richesse en chémorécepteurs serait une adaptation à la diversité chimique des plantes et pourrait être commune à tous les coléoptères, qui sont majoritairement phytophages [1]. 


\section{Une boîte à outils génétique complète}

La caractérisation génétique de Tribolium repose sur les efforts d'une communauté de chercheurs dont les principaux instigateurs travaillent aux États-Unis et en Allemagne. C'est grâce à l'impulsion d'Alexander Sokoloff, dans les années 1960, que les premiers mutants ont été obtenus et la première carte génétique établie [3]. Au début des années 1990, Tribolium devint un modèle pour la génétique du développement des insectes, une discipline qui est dominée par la drosophile alors que son développement est pourtant très particulier. Cette nouvelle phase, inspirée par la génétique évolutive du développement («évo-dévo» pour les spécialistes), connaît aujourd'hui son accomplissement avec la mise au point de tous les outils nécessaires à une étude génétique complète (Figure 2).

Comme il convient à un organisme modèle de la génétique, Tribolium est petit, a un cycle court et s'élève facilement. Ce coléoptère se développe en un mois et se reproduit à température ambiante $\left(25-35^{\circ} \mathrm{C}\right)$ dans de la farine contenant $5 \%$ d'extraits de levure, sans eau, avec changement de milieu tous les 3-4 mois. II est possible de déterminer le sexe des adultes ou des pupes et, contrairement à ce qui se passe chez la drosophile, la recombinaison génétique a lieu non seulement dans les gamètes femelles, mais aussi chez le mâle. Tribolium possède neuf paires d'autosomes et une paire de chromosomes sexuels. II n'existe pas de chromosomes polytènes ${ }^{2}$ et des chromosomes balanceurs $^{3}$ sont déjà disponibles pour un tiers du génome, d'autres étant en cours de création. La carte génétique incorpore plus de 400 marqueurs répartis sur les 571 centimorgan des dix groupes de liaison [6].

Les premières mutations isolées chez Tribolium étaient spontanées et affectaient la forme ou la couleur des yeux, du corps et des appendices adultes [3]. Comme chez la mouche, des mutagenèses à l'EMS (éthyl méthyl sulfonate) permettent d'identifier de nouveaux gènes chez Tribolium $[7,8]$. Le projet de mutagenèse par insertion de transposons, baptisé GEKU (Göttingen Erlangen Kansas USDA), a pour objectif l'obtention d'environ 20000 insertions indépendantes [9]. Des lignées transgéniques de Tribolium sont obtenues en utilisant les éléments mobiles piggyBac ou Minos, avec une efficacité de 20 à $60 \%$ des individus injectés, selon la taille du transgène [10-12]. Ces transposons ont des sites d'insertion différents et peuvent être remobilisés chez Tribolium. Les applications de la transgenèse sont multiples : mutagenèse par insertion, enhancer trap [9], dissection de promoteurs [13]. Les outils UAS/GAL4 ${ }^{4}$ et FLP/FRT 5 sont en cours de développement.

\footnotetext{
${ }^{2}$ Chromosomes géants composés de fragments d’ADN qui ne se séparent pas et qui sont formés de plusieurs brins. Ils sont présents dans les glandes salivaires de larves de diptères dont celles de la drosophile.

${ }^{3}$ Chromosomes construits par mutagenèse, dont la structure cytogénétique est très remaniée par rapport à celle d'un chromosome normal, par exemple pour inhiber des crossing-over. Grâce aux chromosomes balanceurs, des mutations létales et stérilisantes de la drosophile ont pu être maintenues, ce qui a permis de déterminer de nombreuses fonctions essentielles pour l'animal.

${ }^{4}$ GAL4 est un activateur de transcription de levure qui agit après s'être fixé sur des séquences d'ADN spécifiques appelées UAS. La méthode implique le croisement de deux animaux transgéniques. Un transgène exprimant GAL4 sous le contrôle d'un enhancer spécifique de tissu va diriger l'expression d'un gène d'intérêt placé derrière les séquences UAS de réponse à GAL4 dans un second transgène.

${ }^{5}$ Méthode utilisée pour la recombinaison, qui utilise une flippase (FLP) recombinase, dérivée de la levure S. cerevisiae. La FLP reconnaît une paire de séquences cibles, les FRT (FLP recombinase target) flanquant une région génomique d'intérêt.
}

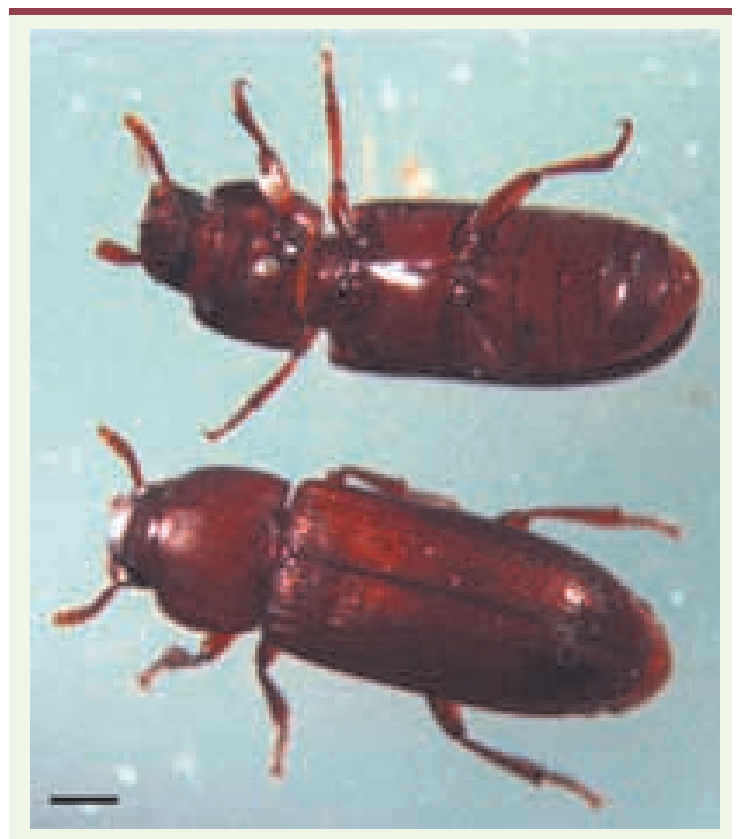

Figure 1. Tribolium castaneum adultes, au corps allongé couleur de châtaigne. Barre : 0,5 mm.

Un avantage majeur de Tribolium est la facilité d'obtention de phénocopies ${ }^{6}$ par injection d'ARN double brin. L'ARN interférant (ARNi) peut être injecté dans l'embryon [14], dans la larve [15] ou dans la pupe [16]. Dans ce dernier cas, cela produit une interférence parentale sur la descendance. Chez cet insecte, comme chez le nématode Caenorhabditis elegans, l'interférence ARN est systémique, c'est-à-dire que les gènes ciblés sont inactivés dans tout l'organisme, quels que soient le type cellulaire et le stade de développement. Cette réponse systémique étant absente ou très limitée chez la drosophile, Tribolium permet d'étudier ce phénomène chez les insectes $[17,18]$.

\section{Tribolium et drosophile : le couple idéal de la biologie du développement des insectes}

Même si la drosophile est un incontournable modèle de la génétique du développement, elle présente des spécificités qui limitent la portée de certaines découvertes [19]. En effet, pour des raisons inconnues, les diptères (mouches, moustiques) ont évolué très vite, ce qui a abouti à des modes de développement et à des génomes différents de ceux des autres insectes. La première révélation apportée par la caractérisation du génome de Tribolium fut de montrer que le groupe ${ }^{6}$ Phénocopie : phénotype réversible d'une cellule ou d'un organisme imitant le phé-
notype résultant d'une modification génotypique. 


\begin{tabular}{|c|c|c|}
\hline & Drosophila melanogaster & Tribolium castaneum \\
\hline Description & Johann Wilhelm Meigen, 1830 & Johann Friedrich Herbst, 1797 \\
\hline Lieu & Museum de Paris & Museum de Berlin \\
\hline Systématique & $\begin{array}{l}\text { Hexapoda, holometabola } \\
\text { Ordre: Diptera } \\
\text { Famille: Drosophilidae }\end{array}$ & $\begin{array}{l}\text { Hexapoda, holometabola } \\
\text { Ordre: Coleoptera } \\
\text { Famille: Tenebrionidae }\end{array}$ \\
\hline Ovaires & $\begin{array}{l}\text { Méroïstique panotrophique } \\
16 \text { ovarioles / ovaire }\end{array}$ & $\begin{array}{l}\text { Méroïstique télotrophique } \\
5-6 \text { ovarioles / ovaire }\end{array}$ \\
\hline Fécondité & 300 œufs / femelle & 500 œufs / femelle \\
\hline Embryogenèse ${ }^{1}$ & $\begin{array}{l}1 \text { jour } \\
\text { Bandelette germinative longue }\end{array}$ & $\begin{array}{l}3 \text { jours } \\
\text { Bandelette germinative courte }\end{array}$ \\
\hline Larves $^{1}$ & 4 jours $^{1}, 3$ stades & 25 jours, 5 à 9 stades (jusqu'à 13 ) \\
\hline Métamorphose ${ }^{1}$ & $4-5$ jours $^{1}$ & 6 jours \\
\hline Chromosomes & 4 chromosomes, XY & 10 chromosomes, XY \\
\hline Génome ${ }^{2}$ & $\begin{array}{l}120 \text { euchr }+60 \text { hétérochr }=180 \mathrm{Mb} \\
\text { Séquence : mars } 2000\end{array}$ & $\begin{array}{l}160 \text { euchr + } 44 \text { hétérochr = } 204 \mathrm{Mb} \\
\text { Séquence : avril } 2008\end{array}$ \\
\hline Gènes & $\approx 13000$ & $\approx 16000$ \\
\hline
\end{tabular}

Tableau I. Comparaison de quelques caractéristiques biologiques de la drosophile et de Tribolium. ${ }^{1}$ : durées à $25^{\circ} \mathrm{C}$ pour la drosophile et à $30^{\circ} \mathrm{C}$ pour Tribolium. ${ }^{2}: \mathrm{Mb}$ euchromatine $+\mathrm{Mb}$ hétérochromatine $=\mathrm{Mb}$ total. d'une zone de croissance postérieure: on parle de bandelette germinative de type court. Au début des années 1990, quelques généticiens du développement se demandèrent si la segmentation des insectes à bandelette germinative courte se faisait selon des mécanismes différents de ceux qui sont identifiés chez la drosophile. Tribolium, dont l'embryon est de type court [23], a permis d'aborder cette question [19] (Figure 3). La première approche consista à étudier chez Tribolium des gènes déjà connus pour contrôler la segmentation de la drosophile. Les résultats descriptifs suggéraient une grande conservation de la régulation génétique de la

frère des diptères et des lépidoptères (papillons) était celui des coléoptères et non celui des hyménoptères (abeilles, fourmis), comme l'affirmait le consensus traditionnel [20, 21]. Du coup, sur l'arbre des insectes, Tribolium occupe une branche idéalement située pour tester la représentativité d'un mécanisme découvert chez la drosophile (Figure 2). Les apports de cette position de choix seront illustrés ici par deux processus du développement des insectes: la segmentation et la métamorphose.

\section{Segmentation :}

\section{gènes conservés, fonctions différentes}

Le corps des insectes est arrangé en segments répétés (ou métamères) selon l'axe antéro-postérieur [22]. Le processus de segmentation se fait par la mise en place d'unités métamériques embryonnaires (les parasegments) qui sont déterminées par une cascade de régulation génétique. Les quatorze segments de la mouche se mettent en place simultanément au début de l'embryogenèse : on dit que la bandelette germinative (la partie segmentée de l'embryon, à l'exception des premiers segments de la tête) est de type long. Or, chez la plupart des insectes, seule une minorité des segments est formée avant la gastrulation, tandis que la plupart des segments sont ajoutés progressivement à partir segmentation. Néanmoins, l'analyse fonctionnelle a montré que les gènes de segmentation des classes gap et pair-rule agissent souvent différemment entre les deux espèces. Par exemple, les gènes buttonhead et empty spiracles n'ont aucune fonction gap chez Tribolium, contrairement à ce qui est connu chez la drosophile [24]. D'autres gènes gap, tels que hunchback, krüppel et giant combinent des pertes de segments, comme chez la drosophile, mais aussi des transformations homéotiques qui ne sont jamais observées chez la mouche $[25,26]$. À noter que, concernant krüppel, les résultats reposent non seulement sur des phénocopies en utilisant la technique ARNi mais aussi sur le mutant amorphe jaws, obtenu par mutagenèse EMS [25]. Enfin, il semble que, chez Tribolium, les gènes gap ne régulent pas les gènes de la classe pair-rule. Ainsi, le phénotype gap défini chez la drosophile est probablement spécifique des embryons à longue bandelette germinative. De même, si les gènes pair-rule de Tribolium ont tous une expression typique dans un parasegment sur deux, certains n'ont pas le phénotype attendu. C'est le cas, par exemple, des gènes fushi-tarazu, odd-skipped et runt, dont les phénocopies sévères produisent des embryons non segmentés, mais sans défauts de parité $[27,28]$. Quant au gène hairy, s'il a bien une fonction pair-rule, c'est uniquement dans la tête et pas pour les autres segments [29]. Autre surprise: le gène sloppy-paired a une fonction pair-rule dans les deux espèces, mais elle concerne les parasegments pairs chez Tribolium et les impairs chez la drosophile [28] ! Ces études révèlent donc une flexibilité évolutive importante de la fonction des gènes précoces de la segmentation. $\varepsilon$ n revanche, la mise en place ultérieure des frontières parasegmentaires semble être bien conservée chez tous les arthropodes [30]. 


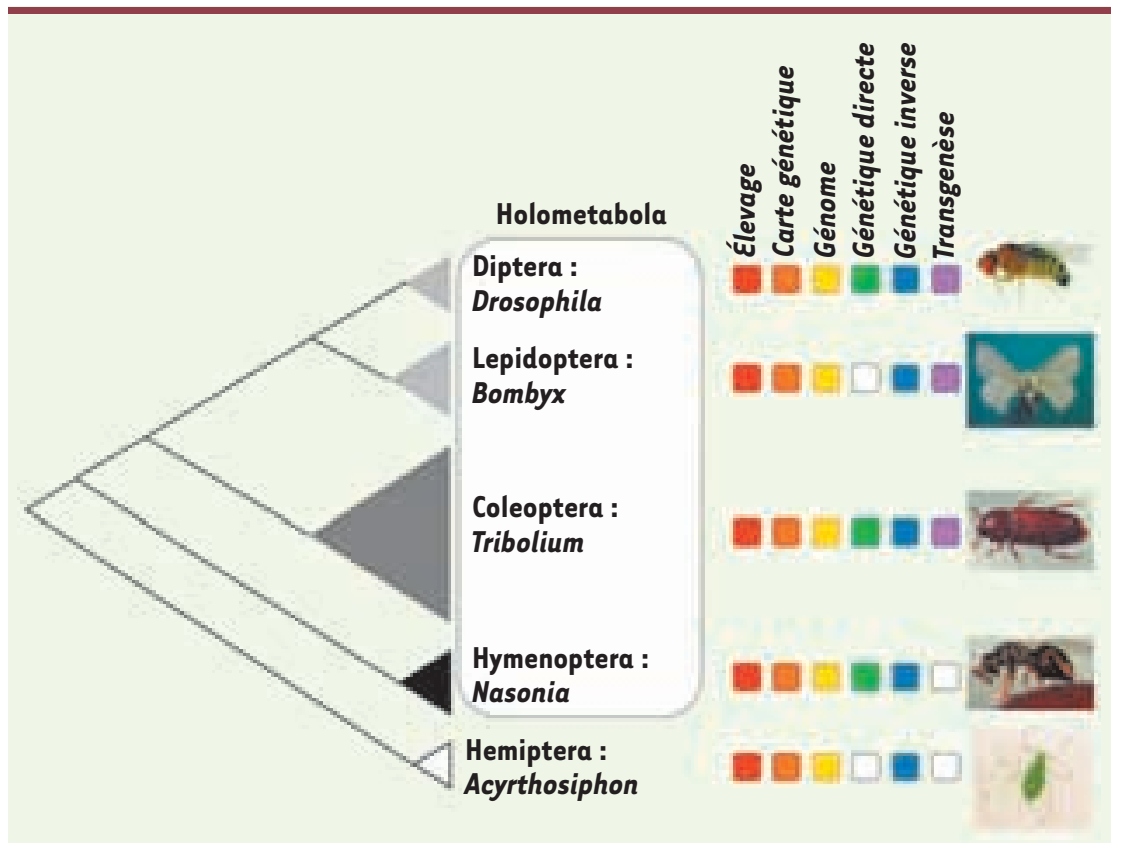

Figure 2. Phylogénie des principales espèces modèles d'insectes en génétique. À gauche, la surface des triangles est proportionnelle au nombre d'espèces de chaque ordre. Les carrés colorés à droite indiquent les outils génétiques disponibles pour chacune des espèces modèles. Un carré pointillé et blanc indique que l'outil n'est pas disponible (dans le cas du Bombyx, environ 400 mutants spontanés existent mais les mutagenèses sont limitées et l'élevage nécessite une infrastructure lourde). À ce jour, seuls Tribolium et la drosophile possèdent une boîte à outils génétique complète et aisément accessible.

Afin de s'affranchir du biais inhérent à une approche de génétique inverse basée sur des gènes candidats déjà connus chez la drosophile, quelques travaux de mutagenèses ont permis d'isoler des mutants qui identifieront peut-être de nouveaux gènes de segmentation chez Tribolium [7, 8]. Par ailleurs, I'utilisation des données d'expression $\mathrm{d}^{\prime} \mathrm{EST}^{7}$ a débouché sur la découverte d'un étrange gène gap appelé mille-pattes dont la perte de fonction conduit au remplacement des segments abdominaux par des segments thoraciques, produisant ainsi des embryons de Tribolium à dix paires de pattes. Contrairement aux autres gènes gap qui codent pour des facteurs de transcription, ce gène produit un $A R N$ polycistronique codant pour quatre peptides, une rareté chez les eucaryotes [31].

Ainsi, certains mécanismes de la segmentation de la drosophile sont probablement spécifiques des embryons à longue bandelette germinative. Auparavant, l'existence de ces mécanismes était souvent extrapolée à l'ensemble des insectes, une vision simpliste qui persiste malheureusement dans de nombreux manuels. Un point crucial est la fréquente absence de corrélation entre l'expression d'un gène et son phénotype mutant chez Tribolium. Ce fait, qui contredit la simplicité du paradigme drosophile, devrait inciter à la plus grande prudence dans l'interprétation des données purement descriptives en évo-dévo. Heureusement, la méthode d'interférence ARN autorise une approche fonctionnelle chez de nombreuses espèces d'insectes.

\section{Le contrôle de la métamorphose}

La croissance des insectes se fait par une succession de mues. Chez les insectes holométaboles, comme la drosophile et Tribolium, les larves grandissent sans changer de morphologie. Cette phase du développement se termine par une métamorphose complète au cours de laquelle

${ }^{7}$ Dans une banque d'ADNc, les EST (expressed sequence tag) correspondent à des fragments d'ADNc qui peuvent être utilisés pour identifier des transcrits et découvrir de nouveaux gènes. se forme l'adulte. Le contrôle temporel de ces changements est assuré par deux hormones dont les rôles sur la morphogenèse sont antagonistes: l'ecdysone la permet alors que l'hormone juvénile l'empêche. II apparaît que la réponse génétique à ces hormones est assez différente entre la mouche et les autres insectes holométaboles.

L'ecdysone est une hormone stéroïde dont le récepteur, $\varepsilon C R$ (ecdysone receptor), hétérodimérise avec un partenaire obligatoire nommé USP (homologue de RXR, retinoid $X$ receptor). Au début de la métamorphose de la drosophile, le complexe ECR-USP régule directement un petit nombre de gènes précoces. Toutes ces protéines sont des facteurs de transcription, dont beaucoup appartiennent à la famille des récepteurs nucléaires. Ces protéines précoces régulent ensuite un grand nombre de gènes tardifs qui sont les effecteurs de la morphogenèse. L'analyse du génome de Tribolium a permis de découvrir que $\varepsilon c R$, usp et une partie des gènes précoces situés au sommet de cette cascade génétique avaient subi une forte accélération évolutive lors de l'émergence du groupe d'insectes auquel appartiennent les diptères et les lépidoptères [32, 33] (Figure 4). Ces protéines ont tellement divergé que la généralisation de certains mécanismes moléculaires identifiés chez la drosophile est impossible. Par exemple, chez la mouche et chez un papillon, la protéine USP possède une large poche contenant un acide gras. Mais la structure de I'hétérodimère $\varepsilon C R-U S P$ de Tribolium a révélé que la protéine USP de cet insecte était un récepteur orphelin, car il n'a pas de poche permettant de recevoir un ligand [34]. De plus, la surface d'interaction entre 


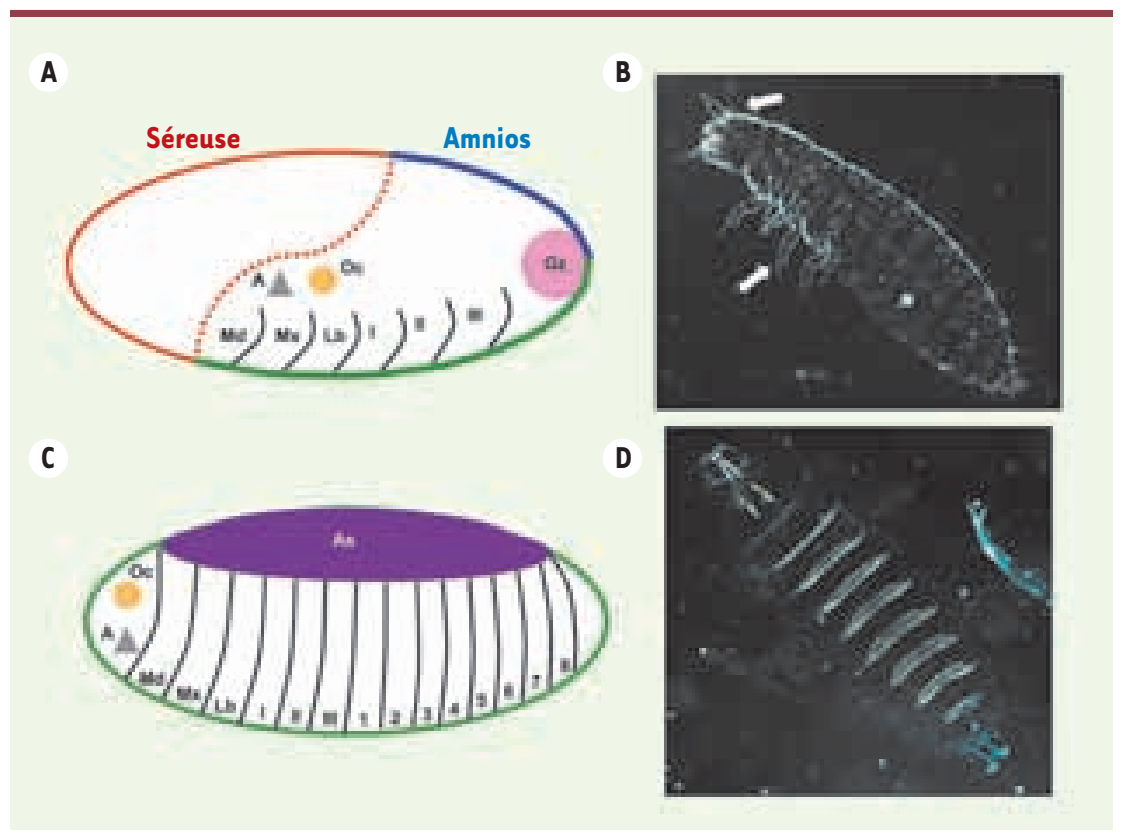

Figure 3. Développements embryonnaires de Tribolium et de la drosophile. Carte des territoires présomptifs au stade blastoderme de Tribolium, courte bandelette germinative (A) et de la drosophile, longue bandelette germinative (C). La partie embryonnaire est en vert, la séreuse en rouge et l'amnios en bleu; chez la drosophile l'amnios et la séreuse sont réunis en une amnioséreuse (As) [38]. Cuticules en fin d'embryogenèse de Tribolium en vue latérale (B) et de la drosophile en vue ventrale (D). À noter : Ia tête bien développée et les pattes de la larve chez Tribolium (flèches blanches). Sur toutes les images, la partie antérieure est à gauche et la partie dorsale en haut. A : antenne ; $0 \mathrm{c}$ : ocelle; Md : mandibule; $M x$ : mâchoires; Lb : labium ; I, II, III : segments thoraciques; 1 à 8 : segments abdominaux. $\mathrm{Gz}$ : zone de croissance (d'après [19]).

ECR et USP est plus grande chez les mouches et les papillons, suggérant que l'hétérodimérisation ne serait pas obligatoire chez les autres insectes [33]. L'analyse évolutive des structures indique donc qu'il existerait au moins deux types de récepteurs à l'ecdysone et que celui de Tribolium serait représentatif de la majorité des insectes.

Contrairement à l'ecdysone, le récepteur et la voie de signalisation de l'hormone juvénile n'ont toujours pas été identifiés. La génétique de la drosophile est demeurée impuissante car, chez les mouches, l'application exogène de l'hormone juvénile n'a presque aucun effet anti-métamorphique, contrairement à ce que I'on observe chez les autres insectes. De plus, chez la drosophile, le seul mutant tolérant à l'hormone juvénile - qui affecte le gène Met (Methoprene tolerant) - est viable et n'empêche pas la métamorphose, peut-être à cause de la redondance due à un gène paralogue appelé gce (germ-cell expressed). La protéine Met est un régulateur de transcription appartenant à la famille des bHLH-PAS ${ }^{8}$ (comme AHR, le récepteur à la dioxine), dont plusieurs membres sont connus pour interagir avec les récepteurs nucléaires qui contrôlent la réponse à l'ecdysone. Des études récentes ont montré que Tribolium possède deux avantages permettant d'aller plus loin. En effet, Tribolium présente une réponse conventionnelle à l'application d'hormone juvénile. De plus, son génome ne contient qu'un seul gène de type Met, et non deux comme chez la mouche. Ainsi, la perte de fonction du gène Met rend Tribolium résistant à l'hor-

${ }^{8}$ bHLH-PAS : Per-Arnt/AhR-Sim basic helix-loop-helix. mone juvénile et induit une métamorphose précoce [35]. Ce résultat est identique à ce qui est observé chez d'autres insectes après ablation des corps allates, les glandes endocrines qui produisent l'hormone juvénile. Ainsi, la protéine codée par le gène Met constitue un très bon candidat pour être le récepteur à l'hormone juvénile. Si cette hypothèse se vérifiait, alors ce serait la plus belle illustration de la complémentarité entre Tribolium et la drosophile.

\section{Une complémentarité utile}

«Biologists tend to be rather conservative in the use of laboratory animals » Alexander Sokoloff, 1966

Les généticiens du développement ont un goût immodéré pour la drosophile. Heureusement, l'évo-dévo réintroduit une certaine biodiversité dans les laboratoires [36]. Que gagneraient les drosophilistes à utiliser Tribolium à côté de leur mouche favorite?

Tout d'abord, Tribolium permet d'étudier des gènes ou des processus qui sont spécifiquement absents ou très divergents chez la drosophile. En plus des exemples vus précédemment, signalons également que la simplification morphologique et l'invagination de la tête embryonnaire de la mouche rendent difficile l'étude de sa segmentation. Au contraire, la présence d'une tête complète chez la larve de Tribolium permet de mieux comprendre le développement de cette partie du corps des insectes [37] (Figure 3). Un autre exemple de réduction extrême d'un organe concerne la fusion de l'amnios et de la séreuse chez la mouche, alors que ces deux annexes embryonnaires sont séparées chez les autres insectes. Là encore, Tribolium permet d'avoir une compréhension plus générale du développement [38] (Figure 3).

Enfin, l'homme et Tribolium partagent environ 560 gènes qui sont absents chez la drosophile [1]. Comme la mouche est utilisée pour comprendre les bases moléculaires et cellulaires de maladies humaines, Tribolium pourrait constituer un modèle alternatif dans certains 


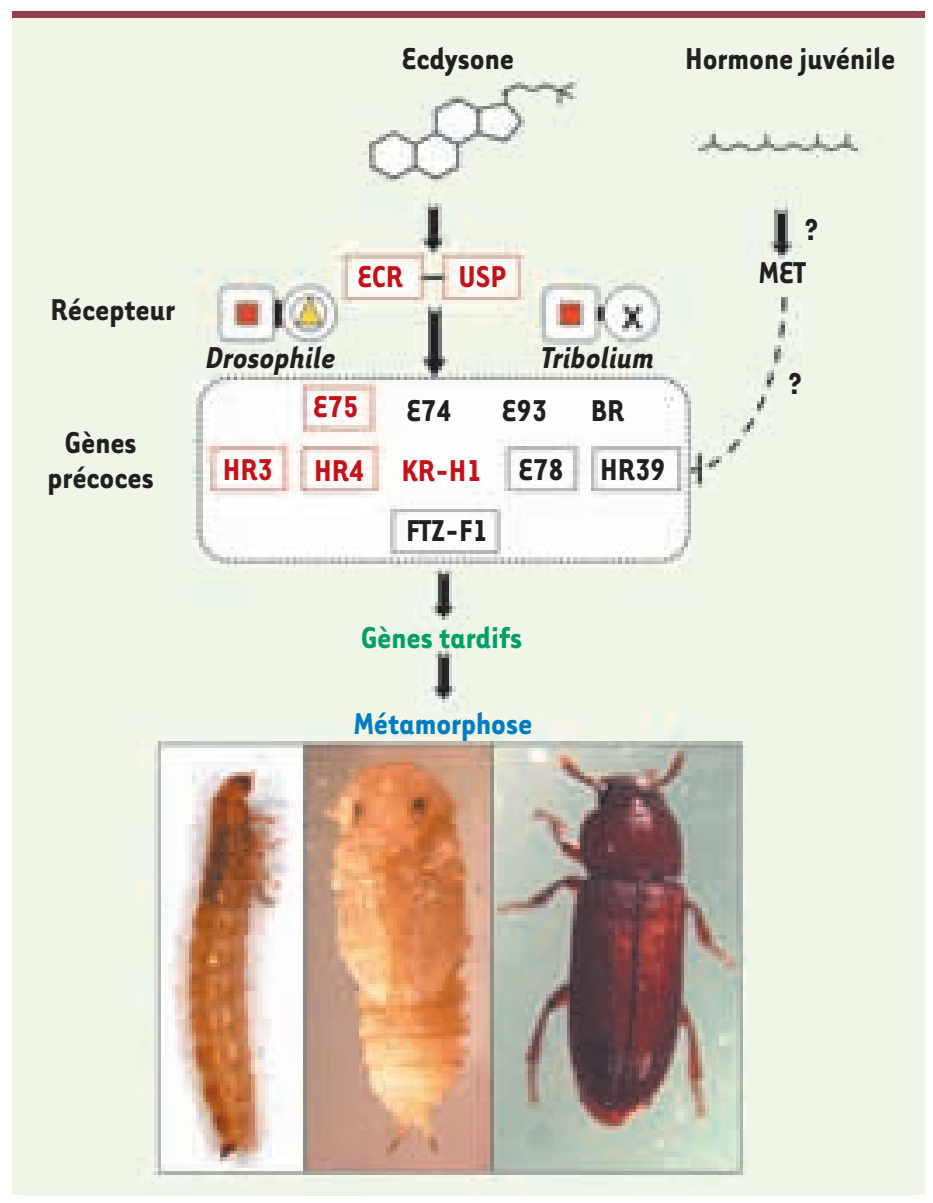

cas. Parmi les gènes perdus chez la drosophile se trouvent ceux qui codent pour la vasopressine et pour son récepteur de la famille des GPCR (récepteurs couplés aux protéines G) [39]. La fonction de la vasopressine, une neuro-hormone peptidique connue pour son rôle diurétique chez les mammifères, est conservée chez Tribolium [40]. Il est probable que cette hormone contribue à la remarquable adaptation des Ténébrionidés à la vie en milieu aride. Signalons enfin que, parmi les gènes perdus par les diptères se trouve la protéine TERT (telomerase reverse transcriptase) qui est la sous-unité catalytique de la télomérase. La baisse d'activité de la télomérase contribue à la sénescence, tandis que sa surexpression est associée à l'immortalisation des cellules cancéreuses. La première structure complète à haute résolution de TERT a été obtenue récemment à partir de la protéine de Tribolium, offrant ainsi une avancée décisive dans la compréhension de cette enzyme essentielle [41].

L'avènement du Tribolium rouge de la farine en tant qu'organisme modèle de la génétique confirme que les biologistes sont bien capables d'innover dans leurs choix d'animaux de laboratoire. $\diamond$

\section{SUMMARY}

When Tribolium complements the genetics of Drosophila

With its recently sequenced genome, the red flour beetle Tribolium castaneum became one of the few model organisms with all the main genetic tools. As a coleoptera, it belongs to the most species-rich
Figure 4. Contrôle de la métamorphose. En haut, les deux hormones principales, ecdysone et hormone juvénile. Le récepteur de l'ecdysone est I'hétérodimère ECR-USP. Chez la drosophile, USP a un ligand (triangle jaune) inséré dans une large poche et la surface d'hétérodimérisation avec $\varepsilon C R$ est grande (barre noire). Chez Tribolium, USP n'a pas de poche (croix noire) et l'interface de dimérisation avec $\varepsilon C R$ est plus petite [33, 34]. Le récepteur de l'hormone juvénile est inconnu, mais pourrait être la protéine MET [35]. Toutes les protéines indiquées sont des facteurs de transcription, celles qui sont encadrées appartiennent à la famille des récepteurs nucléaires. Les protéines rouges ont subi une accélération évolutive dans la lignée menant aux diptères et aux lépidoptères [32]. En bas, de gauche à droite: larve (vue latérale), nymphe (vue ventrale) et adulte (vue dorsale) de Tribolium.

order of animals. Tribolium is also a worldwide pest for stored dried foods. Regarding developmental biology, Tribolium offers a complementary model to the highly derived Drosophila. For example, the function of many gap and pair-rule segmentation genes is different in both species. These differences reveal the evolutionary plasticity between two modes of development, with a long germ band in fly and a short one in Tribolium. This beetle allowed the identification of a new type of ecdysone receptor for holometabolous insects. Finally, in the search for the juvenile hormone receptor, a crucial result was obtained with experiments that could be performed only with Tribolium, and not with Drosophila. Tribolium, in association with Drosophila, should help to understand the general rules of development in insects. $\diamond$

\section{REMERCIEMENTS}

Je remercie Arnaud Chaumot, Pierre Dourlen, Thomas Iwema, Vincent Laudet, Gabriel Markov ainsi qu'un expert extérieur pour leur lecture critique de ce texte.

\section{CONFLIT D'INTÉRÊTS}

L'auteur déclare n'avoir aucun conflit d'intérêts concernant les données publiées dans cet article.

\section{RÉFÉRENCES}

1. Bonneton F. The beetle by the name of Tribolium typology and etymology of Tribolium castaneum Herbst, 1797. Introduction. Insect Biochem Mol Biol 2008 ; 38 : 377-9.

2. Richards S, Gibbs RA, Weinstock GM, et al. The genome of the model beetle and pest Tribolium castaneum. Nature $2008 ; 452$ : 949-55.

3. Sokoloff A. The genetics of Tribolium and related species. New York: Academic Press, 1966

4. Angelini DR, Jockusch EL. Relationships among pest flour beetles of the genus Tribolium (Tenebrionidae) inferred from multiple molecular markers. Mol Phylogenet Evol 2008 ; 46 : 127-41.

5. Hunt T, Bergsten J, Levkanicova Z, et al. A comprehensive phylogeny of beetles reveals the evolutionary origins of a superradiation. Science $2007 ; 318: 1913-6$. 
6. Lorenzen MD, Doyungan Z, Savard J, et al. Genetic linkage maps of the red flour beetle, Tribolium castaneum, based on bacterial artificial chromosomes and expressed sequence tags. Genetics $2005 ; 170: 741-7$.

7. Sulston IA, Anderson KV. Embryonic patterning mutants in Tribolium castaneum. Development 1996; 122 : 805-14.

8. Maderspacher F, Bucher G, Klingler M. Pair-rule and gap gene mutants in the flour beetle Tribolium castaneum. Dev Genes Evol 1998 ; 208 : 558-68.

9. Trauner J, Schinko J, Lorenzen MD, et al. Large-scale insertional mutagenesis of a coleopteran stored grain pest, the red flour beetle Tribolium castaneum, identifies embryonic lethal mutations and enhancer traps. BMC Biol 2009 $7: 73$.

10. Berghammer AJ, Klingler $M$, Wimmer $\varepsilon A$. A universal marker for transgenic insects. Nature $1999 ; 402: 370-1$.

11. Lorenzen MD, Berghammer AJ, Brown SJ, et al. PiggyBac-mediated germline transformation in the beetle Tribolium castaneum. Insect Mol Biol 2003; $12: 433-40$.

12. Pavlopoulos A, Berghammer AJ, Averof M, Klingler M. Efficient transformation of the beetle Tribolium castaneum using the Minos transposable element: Quantitative and qualitative analysis of genomic integration events. Genetics $2004 ; 167: 737-46$.

13. Eckert C, Aranda M, Wolff C, Tautz D. Separable stripe enhancer elements for the pair-rule gene hairy in the beetle Tribolium. EMBO Rep 2004 ; $5: 638-42$.

14. Brown S, Holtzman S, Kaufman T, Denell R. Characterization of the Tribolium Deformed ortholog and its ability to directly regulate Deformed target genes in the rescue of a Drosophila Deformed null mutant. Dev Genes Evol 1999; $209: 389-98$.

15. Tomoyasu Y, Denell RE. Larval RNAi in Tribolium (Coleoptera) for analyzing adult development. Dev Genes Evol 2004 ; 214 : 575-8.

16. Bucher G, Scholten J, Klingler M. Parental RNAi in Tribolium (Coleoptera). Curr Biol $2002 ; 12$ : R85-6.

17. Tomoyasu Y, Miller SC, Tomita S, et al. Exploring systemic RNA interference in insects: a genome-wide survey for RNAi genes in Tribolium. Genome Biol 2008 ; $9: \mathrm{R} 10$.

18. Miller SC, Brown SJ, Tomoyasu Y. Larval RNAi in Drosophila? Dev Genes Evol $2008 ; 218: 505-10$.

19. Schroder R, Beermann A, Wittkopp N, Lutz R. From development to biodiversityTribolium castaneum, an insect model organism for short germband development. Dev Genes Evol 2008 ; 218 : 119-26.

20. Savard J, Tautz D, Richards S, et al. Phylogenomic analysis reveals bees and wasps (Hymenoptera) at the base of the radiation of Holometabolous insects. Genome Res $2006 ; 16: 1334-8$.

21. Zdobnov EM, Bork P. Quantification of insect genome divergence. Trends Genet $2007 ; 23: 16-20$.

22. Cornec JP, Gilles A. Urbilateria, un être évolué? Med Sci (Paris) 2006 ; $22: 493-501$

23. Handel K, Grunfelder CG, Roth S, Sander K. Tribolium embryogenesis: a SEM study of cell shapes and movements from blastoderm to serosal closure. Dev Genes Evol $2000 ; 210: 167-79$.

24. Schinko JB, Kreuzer N, Offen N, et al. Divergent functions of orthodenticle, empty spiracles and buttonhead in early head patterning of the beetle Tribolium castaneum (Coleoptera). Dev Biol 2008 ; 317 : 600-13.

25. Cerny AC, Bucher G, Schroder R, Klingler M. Breakdown of abdominal patterning in the Tribolium Kruppel mutant jaws. Development $2005 ; 132: 5353-63$.

26. Marques-Souza H, Aranda M, Tautz D. Delimiting the conserved features of hunchback function for the trunk organization of insects. Development 2008 ; $135: 881-8$.

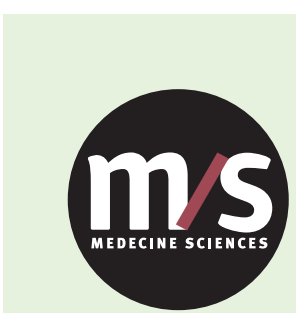

Tarifs d'abonnement M/S - 2010

Abonnez-vous

à Médecine/Sciences
27. Choe CP, Miller SC, Brown SJ. A pair-rule gene circuit defines segments sequentially in the shortgerm insect Tribolium castaneum. Proc Natl Acad Sci USA $2006 ; 103: 6560-4$.

28. Choe CP, Brown SJ. Evolutionary flexibility of pair-rule patterning revealed by functional analysis of secondary pair-rule genes, paired and sloppy-paired in the short-germ insect, Tribolium castaneum. Dev Biol 2007 ; $302: 281-94$.

29. Aranda M, Marques-Souza H, Bayer T, Tautz D. The role of the segmentation gene hairy in Tribolium. Dev Genes Evol $2008 ; 218: 465-77$.

30. Damen WG. Evolutionary conservation and divergence of the segmentation process in arthropods. Dev Dyn 2007 ; 236 : 1379-91.

31. Savard J, Marques-Souza H, Aranda M, Tautz D. A segmentation gene in Tribolium produces a polycistronic mRNA that codes for multiple conserved peptides. Cell 2006 ; 126: 559-69.

32. Bonneton F, Chaumot A, Laudet V. Annotation of Tribolium nuclear receptors reveals an increase in evolutionary rate of a network controlling the ecdysone cascade. Insect Biochem Mol Biol 2008; $38: 416-29$.

33. Iwema T, Chaumot A, Studer RA, et al. Structural and evolutionary innovation of the heterodimerisation interface between USP and the ecdysone receptor $\varepsilon C R$ in insects. Mol Biol Evol $2009 ; 26: 753-68$.

34. Iwema T, Billas IM, Beck Y, et al. Structural and functional characterization of a novel type of ligand-independent RXR-USP receptor. EMBO J 2007 ; 26 : 3770-82.

35. Konopova $B$, Jindra $M$. Juvenile hormone resistance gene Methoprene-tolerant controls entry into metamorphosis in the beetle Tribolium castaneum. Proc Natl Acad Sci USA 2007 ; 104 : 10488-93.

36. Sommer RJ. The future of evo-devo: model systems and evolutionary theory. Nat Rev Genet 2009 ; $10: 416-22$.

37. Bucher G, Wimmer $\varepsilon A$. Beetle a-head. Investigating embryonic head formation using a novel model organism. BIF Futura $2005 ; 20$ : 164-9.

38. Van der Zee M, Berns N, Roth S. Distinct functions of the Tribolium zerknullt genes in serosa specification and dorsal closure. Curr Biol $2005 ; 15: 624-36$.

39. Li B, Predel R, Neupert S, et al. Genomics, transcriptomics, and peptidomics of neuropeptides and protein hormones in the red flour beetle Tribolium castaneum. Genome Res 2008 ; 18: 113-22.

40. Aikins MJ, Schooley DA, Begum K, et al. Vasopressin-like peptide and its receptor function in an indirect diuretic signaling pathway in the red flour beetle. Insect Biochem Mol Biol 2008 ; $38: 740-8$.

41. Gillis AJ, Schuller AP, Skordalakes $\varepsilon$. Structure of the Tribolium castaneum telomerase catalytic subunit TERT. Nature $2008 ; 455: 633-7$.

Deux revues ont consacré un numéro spécial au génome de Tribolium : Dev Genes Evol 2008 ; 218 (3-4).

Insect Biochem Mol Biol 2008 ; 38 (4).

Sites internet :

Beetlebase, Tribolium Genome Database : http://beetlebase.org/

Tribolium group Göttingen : http://wwwuser.gwdg.de/ gbucherl/index.html

US Department of Agriculture, Research Service, Tribolium Genetics, Richard Beeman :

http://www.ars.usda.gov/Research/docs.htm?docid $=12890$
$>$ Grâce à $m / s$, vous vivez en direct les progrès des sciences biologiques et médicales

Bulletin d'abonnement page 314 dans ce numéro de $\mathrm{m} / \mathrm{s}$
TIRÉS À PART

F. Bonneton

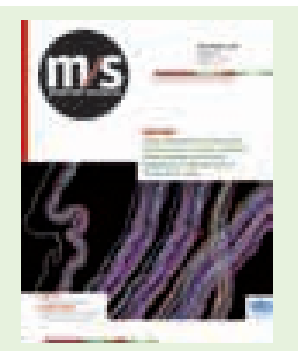




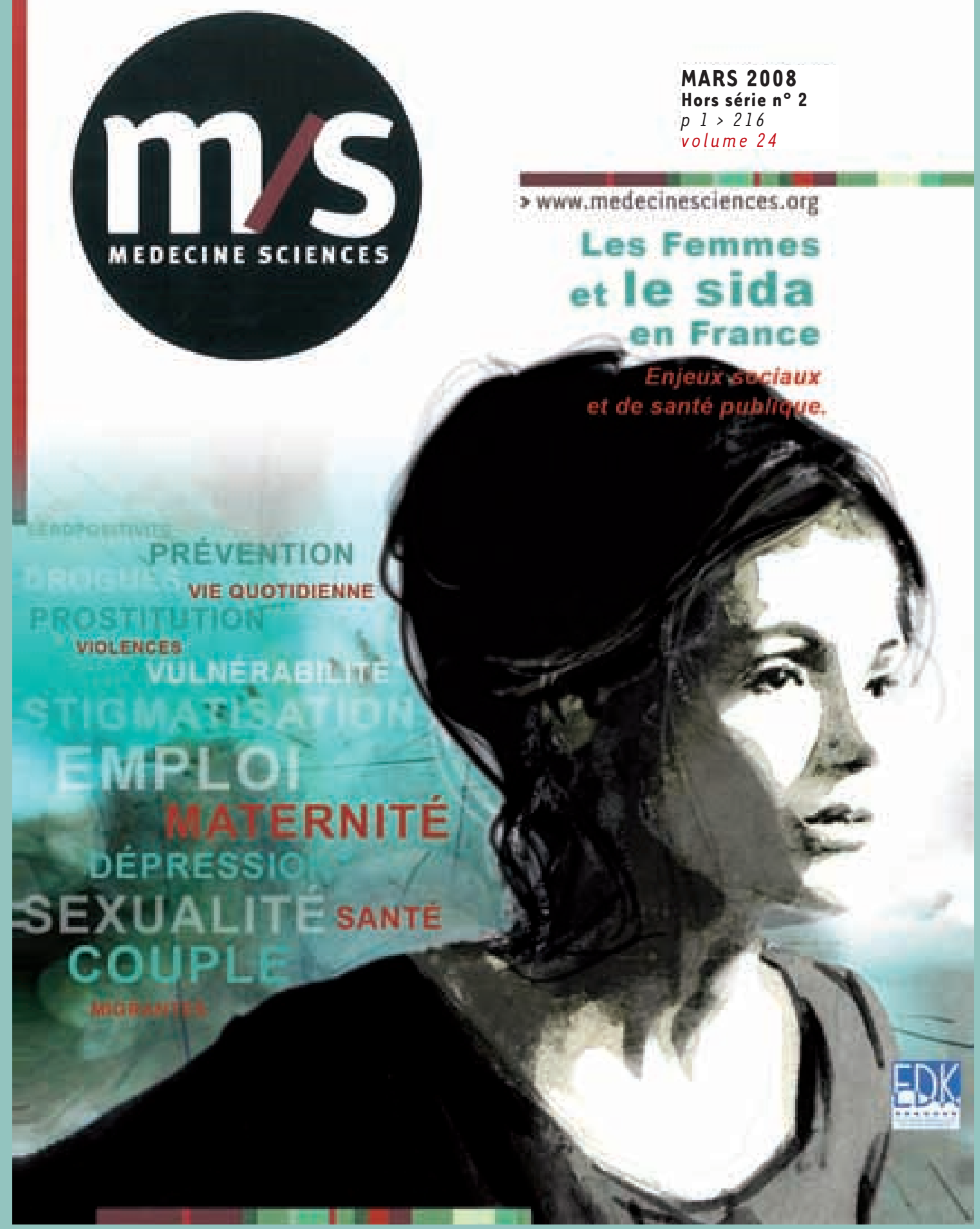

L'Anrs (Agence nationale de recherches sur le sida et les hépatites virales) publie « Les femmes et le sida en France - Enjeux sociaux et de santé publique ».

Un numéro indispensable pour comprendre les nouveaux enjeux de prévention dans le contexte de féminisation et de précarisation de l'épidémie et au moment où se pose la question des conditions de vie des femmes atteintes par l'infection du VIH en France.

Un ouvrage précieux, destiné aux chercheurs, élus, décideurs en santé et représentants associatifs.

\section{Bon de commande}

À retourner à EDK, 2, rue Troyon - 92316 Sèvres Cedex

Tél. : 0155641393 - Fax : 0155641394 - E-mail : edk@edk.fr

NOM :

Prénom :

Adresse :

Code postal :

Ville :

Pays :

Fonction :

Je souhaite recevoir le hors série $n^{\circ} 2$ de $M / S$ Les femmes et le Sida en France: $18 €+3 €$ de port $=\mathbf{2 1} €$ TTC

en ............... exemplaire, soit un total de $€$

$\square$ Par chèque, à l'ordre de $\mathbf{E} \mathbf{D} \mathbf{K}$

$\square$ Par carte bancaire : $\quad \square$ Visa $\square$ Eurocard/Mastercard

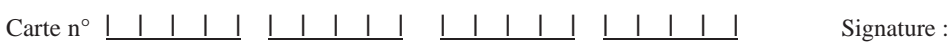

Date d'expiration: $\quad|\quad| \quad|\quad| \quad \mid$

$\mathrm{N}^{\circ}$ de contrôle au dos de la carte : $\quad \leq \quad$ । $\quad$ । 